\title{
SOBRE EL INDIVIDUALISMO METODOLÓGICO. UN PANORAMA ACTUALIZADO DE CUESTIONES Y PUNTOS DE VISTA SOBRE UN VIEJO ASUNTO
}

\author{
Juan Carlos GARCIA-Bermejo OCHOA \\ Universidad Autónoma de Madrid
}

\begin{abstract}
RESUMEN. El individualismo metodológico es y sigue siendo un rasgo característico dela teoría económica. Sin embargo, sus versiones más conocidas son bastante anteriores a los nuevos desarrollos teóricos del último tercio del siglo pasado. El artículo pretende ofrecer un panorama actualizado del tema, teniendo en cuenta esos desarrollos.
\end{abstract}

ABSTRACT. Methodological individualism continues to be a distinctive feature of economic theory. However, most accounts predate the theoretical developments that took pla$c e$ in the last quarter of the $20^{\text {th }}$ century. This paper provides an updated state of the art with a critical discussion of these latest contributions.

\section{Presentación}

En la edición de julio de 2003 del seminario internacional que la Fundación Urrutia Elejalde organiza cada verano, Rachel Kranton presentó el modelo que George Akerlof y ella misma han elaborado sobre el sentimiento de identidad (desde ahora "el modelo A-K»), destacando que si se tiene en cuenta ese sentimiento, se puede llegar a conclusiones y previsiones muy diferentes a las obtenidas por el análisis económico de corte más convencional ${ }^{1}$.

1 En Akerlof (1984: 1-6) puede encontrarse una defensa general de estas líneas de investigación que incorporan adicionalmente factores explicativos de índole psicológico y social. 
El foco del ejercicio, que es a su vez el fenómeno principal que Akerlof y Kranton tratan de modelar, es la forma en la que las preferencias y, en consecuencia, las decisiones individuales pueden verse condicionadas y alteradas por el sentimiento de identidad basado en grupos o categorías sociales.

Huelga decir que el modelo, cuya versión básica es un juego no cooperativo muy sencillo, presenta toda la apariencia de un modelo teórico al uso, eso sí y como es proverbial tratándose de George Akerlof, extremadamente simplificado. Sin embargo, la dependencia social de los comportamientos individuales es una de las tesis básicas de la postura holista (cf., por ejemplo, Viskovatoff 1998: 229). Además, el sentimiento de identidad propicia afirmaciones y actitudes típicamente colectivistas en las que se mantiene que algún pueblo, alguna etnia, algún país o alguna nación determinada es, como entidad colectiva, sujeto de ciertos objetivos, de cierto destino o de ciertos derechos. En consecuencia, cabría preguntarse si el modelo mencionado resultaría ser, al menos parcialmente, de carácter holista en lugar de individualista.

Aun a riesgo de que la ponencia se convirtiera en un simple recordatorio de asuntos y posiciones ya conocidas, la celebración en Buenos Aires en octubre del mismo año del cuarto simposio de la Sociedad Iberoamericana de Metodología Económica invitaba a plantear en él esa pregunta, aunque fuese como un pretexto para suscitar con ello una reflexión y eventualmente un debate sobre cómo pueda percibirse y, en su caso, replantearse desde una perspectiva actualizada el viejo tema del individualismo metodológico (desde ahora "IM").

\section{2. ¿Qué es y qué afirma el individualismo metodológico? La versión de John Watkins y otros aspectos de carácter general}

\section{Algunos apuntes históricos}

Tradicionalmente y con las salvedades y cautelas impuestas en el territorio macroeconómico, la economía ha adoptado una perspectiva individualista, que ha sido considerada uno de los rasgos metódicos tradicionales más peculiares de la disciplina ${ }^{2}$.

\footnotetext{
2 Sobre este tema, puede verse la monografía de John Davis (2004).
} 
Aunque sólo sea por razones de claridad expositiva, conviene que distingamos la perspectiva o el método individualista que recorre la historia de la economía, de la doctrina o las doctrinas que se presentan como una elaboración de esa misma perspectiva. En este sentido, la doctrina clásica que populariza la denominación «individualismo metodológico" es la desarrollada principalmente en el seno de la escuela austríaca, siendo Ludwig von Mises y Friedrich Hayek sus principales autores. Previamente, Carl Menger había realizado una elaboración incial al hilo de su polémica con Gustav Schmöller, en el episodio central de lo que se conoce como la Methodenstreit (cf. Hoover 2000a: 62; Hands 2001: 4347; Arrow 1994: 2; Nagel 1961: 543). En cuanto a los precedentes inmediatos, Mark Blaug (1992: 69-70) afirma que Schumpeter fue el creador de la expresión "individualismo metodológico" al distinguirlo del "individualismo político", $y$ Joseph Agassi (1975: 145) señala que von Mises adoptó la expresión a partir de las expresiones weberianas "individualismo de método" o "metodología individualista». Entre los filósofos de la ciencia, es Karl Popper la figura con la que se suele asociar esa doctrina, quien habría estado especialmente influído por Hayek a este respecto (Blaug 1992: 70).

El IM no se circunscribe a la economía. Pretende prescribir cómo deben ser y deben construirse la historia y las ciencias sociales en general. Su adversario teórico-político principal es el materialismo histórico marxista y su concepción de la evolución histórica basada en la lucha de clases. Aunque la pérdida de influencia del pensamiento marxista tendiera a marginar la apelación al IM, esta apelación se ha reavivado a propósito de la propuesta de autores como John E. Roemer y Jon Elster, conocida como "marxismo analítico" y que proclama la necesidad de aplicar la teoría de la decisión y la teoría de juegos al estudio de temas y problemas tradicionales en el marco del pensamiento y de la ciencia social de orientación marxista (cf. Roemer 1982; Elster 1982).

En relación con la economía, las referencias al IM, o a la perspectiva individualista simplemente, se reavivaron con motivo de la influencia de la macroeconomía keynesiana (en su versión IS-LM), y posteriormente, a propósito de la fundamentación microeconómica de la macroeconomía mediante modelos basados en la conducta de agentes representativos propugnada por la nueva economía clásica (cf. Hoover 2001 ,a: 59-72). 


\section{El IM según J. W. N. Watkins}

Harold Kincaid (1998a: 294) y Maarten Janssen (1998: 4) advierten en una fecha más próxima de lo raras que son las veces en las que el IM está claramente formulado, y de que no existe una definición o una versión generalmente aceptada.

Es cierto que hay una visión básica que, en unos términos u otros, se repite una y otra vez, y que coincidiría con el núcleo de la perspectiva individualista adoptada en la práctica de enfoque microeconómico: la idea de que las explicaciones satisfactorias en las ciencias sociales deben estar basadas en el comportamiento intencional de los individuos. Los problemas y las divergencias se producen cuando se trata de elaborar y desarrollar esa visión básica tan general. Por ello, vamos a recurrir a una versión del IM que ha sido seguramente la referencia más tradicional y frecuente sobre el tema: la elaborada por J. W. N. Watkins.

Uno de los rasgos de originalidad de la presentación del individualismo metodológico a manos de Watkins consiste en plantear el IM como un principio metodológico regulativo, y en ofrecer una caracterización de los principios de esta naturaleza que los distingue de las reglas metodológicas tal como Popper las presenta en su Lógica de la Investigación Cientifica.

En cuanto al contenido del IM, Watkins (1968: 270-1) se expresa como sigue, en un pasaje muy conocido:

De acuerdo con este principio, los constituyentes últimos del mundo social son personas individuales que actúan más o menos apropiadadmente en función de sus disposiciones y de su comprensión de la situación. Toda situación, institución o acontecimiento social complejo es el resultado de una configuración particular de personas individuales, de sus disposiciones, de sus situaciones, de sus creencias, así como de sus recursos físicos y del entorno. Pueden darse explicaciones incompletas o a medio camino de fenómenos sociales de gran escala (por ejemplo, la inflación) en términos de otros fenómenos sociales de gran escala (por ejemplo, el pleno empleo); pero no habremos llegado a las explicaciones más profundas [rock-bottom] de esos fenómenos sociales de gran escala hasta que no hayamos logrado dar cuen- 
ta de ellos deductivamente a partir de enunciados sobre las disposiciones, las creencias y los recursos de las personas individuales, y sobre las interrelaciones entre ellas.

\section{Individualismo ontológico y politico}

Es de notar que, en ese pasaje, el IM es presentado como la yuxtaposición de una teoría metafísica y de un requisito, presuntamente derivado de esa teoría, sobre las explicaciones satisfactorias en las ciencias sociales. Además, el componente metafísico queda subrayado y aclarado al insistir en él de esta manera:

La suposición central de la posición individualista —una suposición que es, justo es admitirlo, contrafáctica y metafísica - es que no existe ninguna tendencia social que no pudiera ser alterada si en relación con las personas individuales interesadas sucedieran dos cosas, que quisieran alterarla y que estuvieran en posesión de la información apropiada. (Watkins 1968: 271)

Todo lo cual se presenta en analogía con el mecanicismo, que es también caracterizado como un principio regulativo, compuesto por una teoría metafísica («los constituyentes últimos del mundo físico son partículas imprenetrables»), y por la postura metodológica de que, en las explicaciones más profundas (rockbottom) de los fenómenos macrofísicos, éstos deben deducirse de enunciados sobre las propiedades de las partículas y las relaciones entre ellas (Watkins 1968: 270). Con ello, Watkins exhibe el tipo de proceder que Blaug (1992: 70) detecta en muchos seguidores de Popper, quienes «deducen el individualismo metodológico de lo que se ha denominado el «individualismo ontológico», es decir, de la proposición de que los individuos crean todas las instituciones sociales y que, por consiguiente, los fenómenos colectivos son simplemente abstracciones hipotéticas derivadas de las decisiones de los individuos».

En desacuerdo con este tipo de planteamiento, Blaug añade la observación, bastante corriente en la literatura, de que el individualismo metodológico es independiente y no se deriva necesariamente del ontológico (cf., por ejemplo, Nagel 1961: 542; Kincaid 1998: 295; Arrow 1994: 1-3). Esa observación, sin embargo, no debe oscurecer que el IM recibe frecuentemente gran parte de su plausibilidad del individualismo ontológico, que, como es también el caso de Blaug, 
parece ser tenido en muchos casos como algo trivialmente obvio (Hoover 2001a: 72-4; Kincaid 1998: 295) ${ }^{3}$.

Análogamente, el individualismo metodológico se asocia también a veces de modo explícito con el individualismo político. Arrow (1994: 2-3), por ejemplo, subraya las actitudes políticas que autores como Hayek y Buchanan consideran asociadas o implicadas por la postura individualista, y señala cómo los austriacos, generalizando la imagen de la "mano invisible" smithiana, asociaron con el individualismo la idea del orden espontáneo, es decir, la idea de que el orden y los cambios sociales son el resultado de las acciones de los individuos que componen la sociedad.

En relación con Popper, Blaug (1992: 69-70) indica que al no establecer de manera nítida la distinción entre el individualismo metodológico y el político, ha favorecido que su defensa del individualismo metodológico, o más bien su crítica de la metodología totalizadora, se utilice a veces como defensa del individualismo político. Blaug piensa que esa utilización es ilegítima, y parece existir un acuerdo tanto o más amplio que en el caso del individualismo ontológico sobre la necesidad de separar el individualismo político del metodológico, y en subrayar la indendencia entre ambos planos (cf. por ejemplo, Arrow 1994: 3).

Una defensa de esta separación e independencia más elaborada de lo corriente se debe a Elster (1982: 453). El epicentro de su postura es la idea de que la ciencia social de inspiración marxista debe adoptar el IM, y sobre todo, hacer uso de teorías como la teoría de la elección racional y la teoría de juegos. Por eso, le resulta fundamental subrayar la independencia del IM respecto de las versiones éticas y políticas del individualismo ${ }^{4}$.

${ }^{3}$ Sensat (1988: 191-94), a quien se debe una de las presentaciones más articuladas del IM, defiende abiertamente que el individualismo ontológico es un componente imprescindible en la articulación del IM por la necesidad de especificar con claridad y detalle los objetos individuales que integran el nivel en el que deben basarse las explicaciones. Kincaid (1998a: 295), por su parte, matiza que el individualismo ontológico parece obvio en sus versiones más triviales y menos informativas, pero que esa apariencia va desapareciendo en cuanto las versiones pretenden ser más elaboradas e informativas. $E$ incluso en sus versiones más simples, puede no resultar tan plausible como parece. Autores como Schumpeter (1967: 888-9) y Hoover (2001a: cap. 3), por ejemplo, rechazan la visión del individualismo ontológico.

4 A veces, además de o en lugar de esta dimensión política, se habla del individualismo ético: véase, por ejemplo, Schwartz 1970: 119 y 123. 
Pero a pesar de las reiteradas declaraciones sobre la independencia lógica del individualismo metodológico del ontológico, y de ambos respecto del político o del ético, resulta, en efecto, difícil evitar la sospecha, cuando no la convicción, de que el componente político fuese una de las motivaciones principales en el caso de von Mises y de Hayek, o incluso del propio Popper, al menos inicialmente (véase, por ejemplo, Schwartz 1970).

Asimismo, si el individualismo metodológico puede parecer más fácilmente independizable del plano político y ético, no lo sería tanto del plano ontológico, al menos para quienes entiendan las teorías científicas bajo una óptica realista. Desde una interpretación de este carácter, no parece fácil defender que las explicaciones individualistas son más satisfactorias si no se comparte de algún modo una visión individualista pre-teórica.

\section{La dimensión explicativa del IM}

Volviendo al IM, éste es presentado habitualmente como un método para desarrollar explicaciones satisfactorias en las ciencias sociales, y es defendido como una estrategia investigadora que garantiza mejor la comprensión de los fenómenos sociales ${ }^{5}$. Sensat (1988: 201) subraya que la visión subyacente al IM sugiere ya que el principal beneficio de esa metodología consistiría en las explicaciones que proporciona.

En ese sentido, el propio Watkins subraya la ventaja que ofrecen las explicaciones individualistas por partir de premisas familiares, casi evidentes, obvias (sobre las disposiciones de los agentes) (Watkins 1968: 279), evidenciando la conexión con la doctrina del Verstehen afirmada por Blaug (1992: 69) y recordando tesis básicas de la tradición apriorista en economía.

En un sentido menos comprometido, Hoover (2001a: 66-7) menciona la parsimonia (la reducción de los fenómenos complejos a un número reducido de principios), aludiendo a continuación, como ejemplos familiares, a la fundamentación mecánica o atomista de la ley de Boyle-Charles mediante la teoría cinética de los gases y a la reducción de los fenómenos macrobiológicos mediante la genética y la química orgánica (Hoover 2001: 66-7).

\footnotetext{
5 Falta nota.
} 
Arrow (1994: 3-4) es también rotundo al respecto, relacionándolo con otro tema frecuentemente mencionado por los individualistas clásicos: la explicación y comprensión de los fenómenos emergentes, de los fenómenos sociales o agregados que son consecuencia no intencionada de las acciones de los individuos. De una manera muy expresiva, al tratar de marcar con nitidez las diferencias entre el IM y dos variantes del individualismo que considera desafortunadas, el psicologismo y la teoría conspirativa de la sociedad, el propio Watkins (1968: 275-8) resalta como una de las tareas más significativas de la ciencia social bajo el IM la de explicar las consecuencias no intencionadas de la interacción de las acciones y reacciones de los individuos, especialmente de las consecuencias perjudiciales, como el paro, cuya explicación más correcta es la que puede llevar a su tratamiento político má adecuado. Y llega a cifrar en esta capacidad del individualismo su mayor rendimiento ${ }^{6}$.

Por su parte, Janssen (1998: 4-6), quien también interpreta el IM como una recomendación sobre las explicaciones en ciencia social, precisamente por ello introduce de manera expresa un requisito de adecuación empírica que, curiosamente, no se suele discutir en la literatura sobre el IM, posiblemente porque se dé por supuesto. Quizá suceda que, dando por supuesta la visión preteórica subyacente, quedaría garantizado a grandes rasgos el cumplimiento de adecuación empírica al proceder tal y como el IM recomienda.

\section{Reducción y reducibilidad en principio}

Otro aspecto que debe ser destacado en la concepción del IM propugnada por Watkins es el papel que en dicho concepto desempeña la reducción. La propia analogía que Watkins (1968: 270) establece entre el IM y el mecanicismo como principios regulativos refuerza este papel, y por influencia de su presentación, lo más habitual en la literatura es encontrar definido el IM en términos reductivos ${ }^{7}$.

${ }^{6}$ También Hayek y Popper expresan la idea de que la explicación de esos fenómenos emergentes es una de las tareas principales de la ciencia social: $c f$. Hands 2001: 46-7; Blaug 1992: 70-1.

${ }^{7}$ Es tan habitual esta forma de presentar el IM que huelga seleccionar citas que la ejemplifiquen. A título anecdótico puede señalarse que en Brodbeck (1968), que es uno de los libros colectivos más conocidos y utilizados en la época sobre la filosofía de las ciencias sociales, los artículos sobre el IM se encuadran en la parte cuarta del libro, que lleva como título Hechos sociales, leyes sociales y reducción. 
En el pasaje de Walkins que recogía su definición del IM, la reducción queda aludida cuando se establece que no se habrá llegado al nivel más profundo de explicación (rock bottom explanations) de los macro-fenómenos (large-scale phenomena) hasta que éstos no sean deducidos a partir de enunciados sobre las disposiciones, las creencias, los recursos de las personas individuales y las interrelaciones entre ellas. En términos literales, pues, se establece un requisito de deducción. El problema, ya destacado por Ernest Nagel (1961:353-4) en su análisis clásico de la reducción, es que para deducir macro-fenómenos a partir de micro-fenómenos puede ocurrir que se necesiten adicionalmente convenciones u otro tipo de condiciones o hipótesis que permitan relacionar los conceptos y términos con los que se describen unos y otros ${ }^{8}$. Por eso, en lugar de hablarse de "deducción" a secas, en la literatura se habla de "reducción", concepto que combina esos dos elementos, es decir, la deducción de la teoría o del fenómeno reducido (en nuestro caso los macro-fenómenos) a partir de la teoría o de los fenómenos reductores, pero llevada a cabo con la ayuda, como premisas adicionales, de enunciados que vinculen los conceptos de uno y de otro nivel, y que pueden incluso establecer el dominio de vigencia de la teoría reducida (cf. Sensat 1988: 191-2; Kincaid 1998: 296) ${ }^{9}$.

No se trata, sin embargo, de una exigencia de reducción sin más, sino de un rquisito más matizado. Según el pasaje de Walkins que transcribíamos más arriba, entre los elementos de los que el científico social individualista debe partir como factores o variables explicativas, podemos distinguir tres grupos: (1) los

${ }^{8}$ Hoover (2001: 66-7) ilustra el asunto con gran claridad valiéndose de la reducción de la ley de Boyle-Charles permitida por la teoría cinética de los gases, que supone a estos efectos que el gas está compuesto de moléculas consideradas como puntos masa perfectamente elásticos a los que se aplica la mecánica newtoniana: «Nótese, también, que las categorías que se aplican a las leyes de Newton y a las leyes de los gases son muy diferentes. Una molécula tiene momento y energía, pero no tiene presión ni temperatura. Para llevar a cabo la tarea de derivación, es necesario identificar propiedades agregadas de los conjuntos de moléculas (su energía y momentos medios) que se corresponderían con las propiedades macro (temperatura y presión) que presentan unas caracteríticas sensibles completamente diferentes. Los fenómenos de la temperatura y de la presión pueden ser considerados como propiedades emergentes de la agregación de moléculas».

9 En este aspecto caben posiciones más o menos rigurosas. Nagel (1961: 542-4), por ejemplo, interpreta que los requisitos impuestos por el IM conllevan la eliminabilidad de los términos y conceptos agregados, lo que requiere un tipo de reducción tan riguroso, basado en la definibilidad explícita de los términos agregados sobre la base de términos individualistas, que se muestra escéptico sobre la posibilidad de que pueda cumplirse en muchos casos. 
factores mentales, psicológicos o subjetivos del agente (disposiciones y creencias), (2) los factores y restricciones físicas (recursos), (3) y los factores sociales o aparentemente sociales (interrelaciones con otros individuos). El problema viene planteado por estos últimos, los factores sociales, que pueden incluso ser determinantes en la formación de los factores mentales de los agentes. Si el IM trata de basar sus explicaciones plenamente satisfactorias de los fenómenos sociales en términos individuales, ¿es legítimo incluir variables explicativas referentes a otros fenómenos sociales?

Dadas las características del modelo A-K, en relación con él cobran un interés particular las cualificaciones que Watkins (1968: 274) introduce a este respecto, al aclarar que el IM admite que la formación de las disposiciones, de las creencias de los individuos y de las situaciones a las que hacen frente puedan explicarse en función de factores sociales. Lo que el IM exige es que uesas explicaciones sean a su vez individualistas [el subrayado es del propio Watkins], explicando esa formación como el resultado de una serie de respuestas conscientes o inconscientes del individuo a su situación cambiante». Más adelante trata de sintetizar: «El individualista metodológico sólo insiste en que el entorno social al que se enfrenta la persona individual, que es el origen de sus frustraciones, y que a veces la manipula y ocasionalmente la destruye, está integrado, si dejamos de lado sus ingredientes físicos, por otras personas, por sus hábitos, su inercia, sus lealtades, sus rivalidades, etc. Lo que el individualista metodológico niega es que la persona individual se vea frustrada, manipulada, destruida o conducida por leyes sociológicas o históricas irreducibles" (Watkins 1968: 278, nota 8).

Como subraya Mongin y se recoge habitualmente en las presentaciones del IM influidas por la de Watkins (cf., por ejemplo, Blaug 1992: 69 y 295; Hausman 1992: 322; Kincaid 1998: 295-7; Sensat 1988: 119), no se trata, pues, de una exigencia de llevar a cabo en cada caso la reducción de los fenómenos sociales a términos individualistas, sino de un atemperado requisito de "reducibilidad en principio» ${ }^{10}$.

${ }^{10}$ Watkins «está dispuesto a aceptar un papel causal para los fenómenos sociales cuando éstos son analizables (en lugar de ser efectivamente analizados) en los términos del individualismo metodológico [...]. De acuerdo con ello, corrijamos la definición inicial y estipulemos que el individualismo psicológico es la doctrina que no admite en principio que los fenómenos sociales formen parte de las variables exógenas. Con lo que queremos decir que los fenómenos sociales que pue- 


\section{El individualismo metodológico en Karl Popper. La racionalidad como caso especial}

Debe apuntarse, por último, una característica importante en el tratamiento del IM por parte de Karl Popper, suscitada por el peso que éste atribuye al método cero o método de la lógica de la situación.

Popper también defiende el individualismo metodológico en su concepción más general, en una línea similar aunque no completamente coincidente con la de Hayek, y en un plano de generalidad similar al adoptado por Watkins. Así, y como recoge Pedro Schwartz (1970: 122), afirma en La Miseria del Historicismo: "La tarea de la ciencia social es la de construir y analizar nuestros conceptos sociológicos cuidadosamente en términos descriptivos o nominalistas, es decir, en términos de individuos, de sus actitudes, esperanzas, relaciones, etc. —un postulado que se podría llamar «individualismo metodológico». Por otra parte, desde muy pronto empezó a cobrar una importancia central en su pensamiento, como el núcleo de las diferencias entre las ciencias naturales y sociales, el método que llamó lógica de la situación o método cero, y que define así:

Me refiero a la posibilidad de adoptar en las ciencias sociales lo que se puede llamar el método de la construcción racional o lógica, o quizá el umétodo cerom. Con esto quiero significar el método de construir un modelo en base a una suposición de completa racionalidad (y quizá también sobre la suposición de que poseen información completa) por parte de todos los individuos implicados, y de estimar luego la desviación de la conducta real de la gente con respecto a la conducta modelo usando esta última como una especie de coordenada cero. (Popper 1957: 170-1) ${ }^{11}$

Como consecuencia de ello y de acuerdo, por ejemplo, con Blaug (1992: 71), el propio Popper mantendría también que el individualismo metodológico debería interpretarse como la aplicación a las cuestiones sociales del "principio de racionalidad", o del "método cero" aplicado a la "lógica de la situación".

den, en principio, ser reducidos no cuentan como fenómenos sociales; lo que hacen más bien es estar en el lugar de las acciones individuales cuyas consecuencias sintetizan, dadas variables causales relevantes" (Mongin, 1998: 46-7).

1 Para más detalles sobre la posición de Popper, puede verse Zaparero 1977. 
Sin embargo y con independencia de la cuestión exegética involucrada en la afirmación de Blaug, conviene distinguir los dos planos puesto que parece haber un acuerdo general en que el enfoque basado en la la hipótesis de racionalidad es un caso especializado de teoría individualista, y en que el IM no presupone la racionalidad del comportamiento individual. Arrow (1994: 4), por ejemplo, se manifiesta en los términos siguientes:

En esta rápida presentación del individualismo, he evitado la expresión "elección racional». El punto de vista individualista es, en principio, compatible con la racionalidad acotada, con la violación de los axiomas de la racionalidad, y con desviaciones en las características de los seres humanos. El paso adicional a la elección racional es, por supuesto, de la mayor importancia práctica para la construcción teórica, pero no es en principio necesario para el punto de vista individualista ${ }^{12}$.

\section{El requisito de reducibilidad a términos individualistas}

\section{Algunas observaciones de carácter general}

No debe sorprender que el requisito de reducibilidad haya sido el aspecto de la versión de J. Watkins cuya pertinencia se haya puesto más en cuestión. La "reducibilidad en principio" es una exigencia matizada y suavizada que, aparentemente al menos, hace del IM una doctrina más aceptable. Sin embargo, esa misma suavización de la postura puede acarrear problemas por sí misma.

Por ejemplo, se ha señalado el regreso al infinito al que puede dar lugar (Mongin 1998: 47; Mäki, apud Backhouse et al. 1998: 57). Si se permite partir de variable explicativas de carácter social con tal de que sean reducibles, esas variable pueden ser explicadas a su vez mediante otras del mismo carácter sin que el proceso tenga por qué tener un final definido.

Más importante, sin embargo, es el problema que se crea sobre cómo decidir si se cumple o no el requisito. La exigencia de tener que reducir a términos individualistas todos los términos agregados, aunque muy restrictiva, tiene la

${ }^{12}$ Puede verse también Janssen 1998: 5 o Hausman 1992: 97. 
ventaja de que ser más clara porque una explicación no resultará aceptable mientras que esa reducción no se haya llevado a cabo. Por el contrario, limitarse a exigir que los factores o fenómenos explicativos de carácter social sean susceptibles de ser explicados en términos individuales, plantea la cuestión de cómo decidir cuándo un factor social es explicable o reducible de esa manera y cuándo no. Un pasaje de Philippe Mongin (1998: 47) es muy elocuente sobre el problema:

¿Cómo sabemos si un fenómeno social es o no reducible en principio? Planteando crudamente la cuestión, sólo podemos responder esta cuestión si tratamos de reducir de hecho el fenómeno, y si tenemos éxito o fracasamos. Como es natural, no es éste el tipo de comprobación que pretendemos aplicar. Tendríamos que ser capaces de resolver la cuestión de la reducibilidad sin tener que tratar de llevar a cabo la reducción. Lo que podría contar en este contexto como garantía sería un bosquejo de una forma de llevar a cabo la reducción, o algún resultado teórico previo del que se desprendiera que la reducción sería realmente posible, o el conocimiento del científico social de que la reducción se habría llevado a cabo bajo circunstancias semejantes. Es delicado establecer criterios, pero son necesarios. En su ausencia, el individualista será propenso a considerar cualquier fenómeno social como reducible «en principio".

Aunque sea de refilón, en ese mismo pasaje de Philippe Mongin se deja ver otro importante problema de ambigüedad, en este caso sobre las restricciones que el requisito de reducibilidad impone a la propia investigación. Imaginemos que, como sucede en el caso del modelo A-K, se introducen factores explicativos de carácter social, y supongamos además que son reducibles a términos individuales, y que no hay dificultad ninguna en decidir o en aceptar que lo son. La explicación aportada en un caso así es como un expediente provisional que no resulta plenamente satisfactorio. ¿Pero qué significa eso? ¿Debemos pasar prioritariamente a intentar reducir a términos individuales los factores sociales introducidos? ¿Podemos orientar el análisis hacia otras cuestiones aunque la explicación aportada no sea plenamente satisfactoria?

Tendríamos cuatro grandes grupos de explicaciones. Uno, el de las explicaciones fundamentales y plenamente satisfactorias por estar formuladas exclusivamente en términos individuales, junto con variables no sociales para describir el entorno en el que se toman las decisiones. El segundo, el de las explicaciones 
también satisfactorias por cuanto incorporan algún factor o fenómeno explicativo de carácter social, pero que está reducido o explicado en términos exclusivamente individualistas en algúna otra explicación o convención. $\mathrm{El}$ tercero, el de las explicaciones mediana o provisionalmente satisfactorias que incorporan algún factor o fenómeno explicativo de carácter social, que es reducible o explicable en términos individuales, pero que no está reducido o explicado por el momento. Por último, el de las explicaciones insatisfactorias por incorporar factores o fenómenos sociales irreductibles.

Esta forma de ver las cosas, parece poder interpretarse como una recomendación a ir convirtiendo las explicaciones medianamente satisfactorias en plenamente satisfactorias, reduciendo o explicando las variables explicativas de carácter social o agregado. Lo que, como subraya Uskali Mäki a propósito del individualismo institucionalista propuesto por J. Agassi, del que nos ocupamos más abajo, introduce una especie de dimensión temporal indeterminada: "A corto plazo, uno acepta las instituciones como dadas, mientras que a largo plazo las explican (Backhouse et al. 1998: 57).

Pero con independencia de esta dimensión, la recomendación de ir completando las explicaciones mediante otras en las que se vayan logrando la reducción de los elementos explicativos de carácter social aducidos por aquellas, ya no sería un requisito sobre explicaciones aisladas. ¿Sería entonces un requisito cuya satisfacción depende del estado de la disciplina? ¿O debería ser satisfecho globalmente por las ciencias sociales? ¿Qué significaría un requisito interdisplinar de este tipo?

El propio modelo de Akerlof y Kranton ilustra que exigir de la propia economía que se ocupe de dar cuenta en términos individuales de la existencia de grupos y categorfas sociales que inducen el sentimiento de identidad puede ser excesivo. A este respecto parece mucho más realista la idea de Joseph A. Schumpeter (1967: 888-9), quien en la cuarta parte de su gran obra sobre la historia del análisis económico, enfoca el individualismo metodológico desde un punto de vista más pragmático y moderno, independizándolo de las pretensiones o requisitos de largo alcance, como la reducibilidad, que presuponen una teoría ontológica o social onmicomprensiva. Así, después de criticar el individualismo social por implicar una teoría de esta naturaleza, se refiere al individualismo metodológico en estos términos: 
Sin embargo, de esto no se sigue que, en aras de los propósitos específicos perseguidos en un conjunto particular de investigaciones, no sea nunca admisible partir del comportamiento dado de las personas individuales sin tener que adentrarse en los factores que hayan dado lugar a ese comportamiento. La conducta en el mercado de una ama de casa puede analizarse sin adentrarse en esos factores. Este tipo de práctica puede venir sugerida por consideraciones de división del trabajo entre las diferentes disciplinas sociales y no requiere ni implica ninguna teoría sobre el tema de la Sociedad o del Individuo [en mayúsculas en el original]. (Schumpeter 1967: 888-9)

El modelo A-K se preocupa por la dependencia social de las preferencias individuales, que se admite previamente como un fenómeno avalado por la evidencia disponible, sin que se aborde en ningún caso la cuestión de "reducir» ulteriormente las variables exógenas no individuales. En el sentido apuntado por Schumpeter, ésta sería una actitud más apropiada en un caso así, en el que no parece demasiado pertinente pasar a ocuparse de si es posible explicar o no desde una perspectiva individualista la formación de los grupos, categorías y diferencias sociales en los que se basa el sentimiento de identidad. En efecto, uno tendería a pensar que ésa sería, en todo caso, una tarea más apropiada de los sociólogos, y en el caso del sentimiento de identidad también de psicólogos, que de economistas. Y eso es precisamente lo que hacen Akerlof y Kranton, dar cuenta de los acuerdos entre sociólogos y psicólogos sobre el tema, sin adentrarse en cuestiones como la posible reducción del sentimiento de identidad y de su formación.

Además, y ésta es una cuestión crucial que Julius Sensat (1988: 201-2) plantea en términos generales, ¿mejoraría la explicación aportada por Akerlof y Kranton cuando se alcanzara la reducción final necesaria para conseguir que aquélla fuese plenamente satisfactoria? ¿Mejoraría el modelo y el mecanismo que incorpora para explicar cómo el sentimiento de identidad puede afectar a las decisiones individuales, si se añadiera una explicación individualista al proceso de formación de las categorías y grupos sociales a los que se adscribe la gente? En analogía a las conclusiones que propone Sensat, esa reducción, caso de ser posible, podría aumentar los elementos de juicio referentes a un proceso más global, pero no parece que pueda afirmarse sin más que fuera a mejorar la explicación que el modelo pretende ofrecer. 
A lo que se puede añadir que, de acuerdo con una idea ampliamente aceptada, la única justificación del IM debe descansar, a la postre, en su rendimiento heurístico y no en razones de principio ni en la aparente plausibilidad de su visión ontológica o sociológica subyacente (cf. Kincaid 1998: 299-300: Hausman 1992: 98).

\section{¿Es la reducibilidad en principio una restricción que se respete en economía?}

Los comentarios anteriores resaltan dificultades a la hora cumplir con el requisito de reducibilidad. En relación con el modelo A-K, limitarse a esos comentarios significaría dar por supuesto que la identidad y los demás elementos de carácter social que introducen los autores son reducibles, y que su reducción a términos individuales no parece constituir una tarea especialmente problemática. Sin embargo, esa reducibilidad no es tan claramente visible ni tan fácilmente realizable, si es que es realmente viable. Lo que, de otro lado, no sería un acontecimiento singular ni peculiar del modelo. También sucedería con otros elementos explicativo aportados por la teoría económica.

\section{Diagnósticos generales}

La economía, o por mejor decir, la microeconomía es considerada por lo general como la instancia especialmente ilustrativa, si no la más, del individualismo metodológico. El requisito de que las explicaciones de los fenómenos económicos proporcionadas por las piezas teóricas estén formuladas y basadas en términos de las decisiones (acciones y reacciones) de los individuos (agentes individuales), y no lo estén en términos de otras categorías sociales, es un requisito vivo y claro de la práctica académica (Arrow 1994: 1). El propio Popper confiesa que su propuesta del método cero era "un intento de generalizar el método de la Teoría Económica de forma que resultase aplicable a otras ciencias sociales" (cf. Blaug 1992: 71; Hands 2001: 284).

Sin embargo, cuando se entra en detalles, las cosas no parecen tan claras, especialmente si la idea del IM incluye el requisito de reducibilidad. Un diagnóstico especialmente autorizado sobre el carácter individualista de la economía es el que lleva a cabo Kenneth J. Arrow (1994). Por un lado y como hemos tenido ocasión de apuntar más arriba, defiende el valor y la vigencia de la perspecti- 
va individualista. Pero la tesis que sostiene como conclusión de su análisis matiza mucho el tipo de perspectiva individualista que se detecta en la economía y que, a su juicio, no se ajustaría a los preceptos de la «reducibilidad». Así, en una frase lacónica, escribe: "De hecho, todo modelo económico en el que uno pueda pensar incluye principios y conceptos sociales irreducibles» (Arrow 1994: 2). Y más extensamente, se manifiesta en los términos siguientes, muy semejantes a las afirmaciones con las que concluye el artículo:

Hoy deseo defender que un examen pormenorizado del análisis económico más convencional muestra que se usan realmente y todo el tiempo categorías sociales y que éstas parecen ser absolutamente necesarias para el análisis, y no meras figuras retóricas que pueden ser eliminadas si es preciso hacerlo. Defenderé adicionalmente que la importancia de la información tecnológica en la economía es un caso especialmente significativo de una categoría irreductiblemente social en el aparato explicativo de la economía. (Arrow 1994: 1)

\section{Una dificultad bastante obvia: la macroeconomía keynesiana y neokeynesiana}

Hay un foco de dificultades para el IM bastante obvio. Los modelos macroeconómicos del tipo IS-LM en los que se fue concretando y desarrollando la influencia de John M. Keynes no se adecuaban al IM, por cuanto no parten expresamente de hipótesis o postulados sobre la conducta de los agentes. $\mathrm{Y}$ algo similar sucede con los modelos neokeynesianos y con los modelos de crecimiento concebidos como sistemas de relaciones funcionales entre macromagnitudes ( $y$ sus tasas de variación). Por ello, Blaug (1992: 71) subraya las «implicaciones devastadoras" que tendría la aplicación estricta del IM al excluir "todas las proposiciones macroeconómicas que no puedan ser reducidas a proposiciones microeconómicas, y puesto que pocas de ellas han sentado sus fundamentos microeconómicos, esto supondría a su vez el decir adiós a casi toda la macroeconomía recibida" ${ }^{13}$.

13 Naturalmente, algo parecido sucede con enfoques distintos a la corriente principal, como la economía evolucionista (Hands 2001: 385), aunque este tipe de conflictos son más de esperar porque el IM es en gran medida una defensa de la perspectiva típica de la corriente (microeconómica) principal y una crítica a enfoques contrapuestos como el marxista, la escuela histórica alemana y parte del institucionalismo. 
En fechas más recientes Kevin Hoover (2001a y b) ha dedicado una especial atención al tema de la reducibilidad de la macroeconomía, en relación con el programa de fundamentación microeconómica de la macroeconomía inspirado principalmente por Robert Lucas y llevado a cabo en la llamada nueva economía clásica y en la escuela de los ciclos reales.

Como Hoover se esfuerza en poner de relieve y como tendremos ocasión de ver más abajo, en ese programa no se trata de reducir la macroeconomía a la microeconomía de la misma manera que la ley de Boyle-Charles es reducida por la teoría cinética de los gases. Lo que se hace es derivar las conclusiones del modelo a partir de las decisiones de agentes representativos, como si la evolución de la economía fuera el resultado directo de esas decisiones. Por ello, por lo que hace a su evaluación de la fundamentación microeconómica de la macroeconomía propugnada por la nueva economía clásica, y en un sentido muy cercano al de la crítica que había formulado Kirman (1992), concluye que la pretendida microfundamentación es algo que no tiene lugar, sería un mero "simulacro", puesto que se apoya en la conducta de agentes representativos inexistentes cuya conducta no está justificada en la de los agentes individuales, por más que sea análoga a la de ellos (Kirman 1992: 83).

Por otra parte, algunos de los argumentos que despliega son aplicables al problema de la reducción de la macroeconomía en general. Destacan dos de ellos, referidos al problema que el autor relaciona con Cournot y el problema de la agregación (de magnitudes).

Hoover (2001a: 70-72) bautiza como "el problema de Cournot» una de las principales razones por las que la macroeconomía no puede ser, como cuestión de hecho, reducida a términos individualistas. Lo formula de esta manera:

Incluso en relación con un volumen de gas relativamente pequeño, está obviamente fuera de toda posibilidad práctica prescindir de toda medida de la temperatura y la presión y disponer, en cambio, de una información precisa sobre las velocidades de cada y de toda molécula. Análogamente, es absurdo pensar que la economía práctica puede disponer de la información sobre las decisiones y las restricciones a las que se enfrenta cada agente individual en la economía. Llamo a esto el problema de Cournot, porque su primera formulación clara se encuentra en sus Investigaciones sobre los Principios Mate- 
máticos de la Teoria de la Riqueza (1838) [...] Como decía anteriormente, nadie cree que los economistas son capaces en la práctica de describir los problemas de decisión de millones de individuos y de agregarlos hasta descubrir el comportamiento macroeconómico resultante.

Además, el problema de Cournot reaparece como un problema al que tienen que enfrentarse los propios agentes reales a la hora de formar sus expectativas económicas. Lo que refuerza la idea de la irreductibilidad del nivel macroeconómico, porque ese problema subsistiría en modelos de enfoque individualista (Hoover 2001 a: 73-4).

En cuanto al problema de la agregación, al que también se refiere Mongin (1998: 51), Hoover (2001a) subraya las dificultades que encierra por las condiciones tan restrictivas que presupone su resolución. Así, después de introducirlo, desarrolla un ejemplo para ilustrar los requisitos exigidos por el llamado teorema delbien compuesto de Hicks, concluyendo de este modo: «Aunque el ejemplo que hemos examinado sea extremadamente simple, aporta un mensaje muy general. Las condiciones requeridas para llevar a acbo un agregación precisa son muy exigentes y prácticamente nunca se cumplen en ninguna instancia práctica» (Hoover, 2001 aa: 79).

\section{¿Es individualista la microeconomía?}

Pero si se pretende apurar la cuestión de hasta qué punto respeta la economía el IM, hay que llevarla al terreno más favorable para la perspectiva individualista. Por eso, la pregunta más significativa es hasta qué punto se ajusta la microeconomía al IM (que hasta el momento estamos considerando en la versión de Watkins).

Tampoco en este terreno las cosas son como parecen. Más arriba veíamos las conclusiones generales que mantiene Arrow (1994), basándose en un análisis en el que pasa revista a teorías y temas concretos. En primer lugar, examina el equilibrio general competitivo, que al constituir la expresión teórica del mito de la mano invisible debería ser uno de los mayores exponentes de la perspectiva individualista. Sin embargo, ya detecta elementos, sobre todo el sistema de precios, a los que considera irreductibles a una explicación individualista. 
Cómo toman sus decisiones individuos y empresas depende de muchos factores: gustos, actitudes frente al riesgo, expectativas sobre el futuro [...] [D]ejo de lado muchas otras cuestiones sobre los papeles e influencias sociales en esa descripción. Los gustos pueden estar causados socialmente; las expectativas están influidas por los demás; las empresas son organizaciones, no individuos [...] [A] pesar de dejar de lado todas estas cuestiones, se mantiene la presencia de un elemento que no es de naturaleza individual: los precios a los que se enfrentan las empresas y los individuos. ¿Qué persona individual ha elegido los precios? Al menos en la teoría formal, ninguno. Quedan determinados en (no por) las instituciones sociales conocidas como mercados, que igualan oferta y demanda. (Arrow 1994: 3-4)

Además, prolonga su análisis para concluir con la inexistencia de un resultado satisfactorio en el que, en el marco de la teoría de juegos, ese sistema de precios sea el resultado de las conductas de los agentes.

El mismo Arrow apunta que el equilibrio competitivo sería, incluso dentro de la economía convencional, el caso más favorable al individualismo, y de todos modos, claramente más favorable que otros apartados de la teoría, como por ejemplo, el dedicado a las externalidades, en muchas de las cuales el efecto final depende de la actuación conjunta de muchos agentes individuales (Arrow 1994: 6). Pero el elemento que Arrow considera más irreductible y en el que fija una mayor atención es el conocimiento, especialmente el conocimiento científico y técnico. Por un lado, ese conocimiento es capital para el funcionamiento de las economías y su crecimiento (Arrow 1994: 6). Y por el otro, la información, y en especial, el conocimiento técnico es una externalidad (ya señalada por Marshall) que tiene a la vez aspectos públicos y privados (Arrow 1994: 8). Además, el fondo de conocimientos existente, y que se va transmitiendo de generación en generación en una sociedad, tiene un marcado carácter social (Arrow 1994: 6). Pero es en el acceso público a la información, una vez que ésta se ha hecho pública, donde Arrow detecta una dimensión irreductiblemente social (una externalidad) del conocimiento técnico por la que la economía no puede ajustarse por completo a la perspectiva individualista (Arrow 1994: 8). Por ello, concluye así su diagnóstico:

Pero concluyo que las variables sociales, no asociadas con ningún individuo particular, son esenciales para estudiar la economía o cualquier otro 
sistema social y que, en concreto, el conocimiento y la información técnica tiene un componente ineludiblemente social, cuya importancia es cada vez mayor. (Arrow 1994: 8)

\section{¿Es individualista la teoría de juegos?}

En el mismo artículo, Arrow también se pregunta hasta qué punto es individualista la teoría de juegos. Por un lado, comienza destacando los rasgos individualistas de la teoría, que considera como "la formulación actual del individualismo metodológico" (Arrow 1994: 4). Pero su examen de la teoría del oligopolio le permite subrayar dos aspectos que avalarían también en este caso la tesis general del artículo, en el sentido de que «la conducta individual está siempre mediatizada por relaciones sociales, que forman parte de la descripción de la realidad tanto como la conducta individual" (Arrow 1994: 5). El primero de estos dos aspectos es el núcleo del problema de las decisiones estratégicas, el problema que tiene cada jugador de conocer la conducta que vayan a seguir los demás, y el consiguiente problema de la consistencia de las expectivas que cada jugador se haya formado a este respecto. El segundo, que ilustra refiriéndose al modelo de Bertrand, es el carácter social de la fijación y el cambio de las reglas de juego.

En cualquier caso, la teoría de juegos trae consigo una variedad de situaciones y conceptos que las versiones clásicas del IM desconocen, y que cualquier versión actualizada debería recoger y reflejar. En este sentido y reflejando una posición muy cercana a la mantenida por Rubinstein (1998), Mongin (1988: 51-52) se expresa en estos términos en relación con los autores clásicos del IM, como Menger, Popper y Hayek:

[T] odos ellos parecen aceptar que el nivel micro puede ser definido independientemente del nivel macro. Según el planteamiento típico, una vez dadas las decisiones micro, el fenómeno macro es el resultado de las consecuencias no propuestas de esas decisiones, que son exógenas respecto del fenómeno macro. Esta suposición puede haber sido apropiada en los contextos históricos y sociológicos en los que estaban interesados los autores mencionados más arriba [...] Pero las situaciones de teoría de juegos son más obstinadas. En ellas, el elemento análogo al agregado social es un resultado de equilibrio del juego, las elecciones individuales son elecciones de estrategias, y sabemos que los equilibrios y las estrategias deben relacionarse entre ellos interna- 
mente, no externamente. La circularidad entre los niveles micro y macro es de alguna manera de lo que trata la teoría de juegos. Constituye, o al menos es lo que propongo, una lección importante, casi filosófica, del amplio trabajo llevado a cabo sobre el equilibrio de Nash que no se puede separar fácilmente la noción "micro" de un agente individual racional de la noción «macro» del resultado de equilibrio del juego.

\section{La versión propuesta por Joseph agassi y la determinación por sobrevenida}

Joseph Agassi (1975) presenta una nueva propuesta, que goza también de una amplia difusión y en la que desaparecen los problemas planteados por el requisito de la reducibilidad en principio. Esos mismos problemas han favorecido, asimismo, que se haya prestado una atención creciente al concepto de la determinación por sobrevenida (supervenience), al que dedicaremos unos breves comentarios posteriormente.

\section{La versión de Joseph Agassi del individualismo metodológico. El individualismo institucionalista}

El objetivo de Agassi es presentar una nueva modalidad de individualismo, que apellida «institucionalista», que sería una vía intermedia entre el psicologismo y el holismo, y que resulta ser notablemente más flexible y operativa que la versión reduccionista del IM. Pero sobre todo, logra centrar la contraposición entre el individualismo y el holismo en un criterio claro, sencillo y definido, $y$ que, según Mongin (1988: 44), es el mejor de los disponibles.

\section{Las contraposiciones de partida}

En su exposición, Agassi se sirve de dos contraposiciones. Una, la que media entre el individualismo, por un lado, y el holismo o colectivismo, por el otro. La segunda distinción es la que separa el psicologismo del institucionalismo.

Para ilustrar el contenido de cada contraposición, asocia con cada una de las cuatro posturas tres proposiciones típicas o peculiares. Por ejemplo, en relación con el individualismo y el holismo, esas tres afirmaciones son las siguientes, respectivamente: 
Holismo o colectivismo

(1.a).-La sociedad es el "todo", que es más que sus partes (holismo).

(2.a).-La "sociedad" afecta los objetivos de los individuos (colectivismo).

(3.a).-La organización social influencia y constriñe la conducta de los individuos (análisis institucional).

Individualismo

(1.b).-Los individuos son los únicos que tienen objetivos e intereses (individualismo).

(2.b).-El individuo se comporta de acuerdo con su objetivo, dadas sus circunstancias (principio de racionalidad).

(3.b).-La acción de los individuos puede cambiar la organización social (reforma institucional) ${ }^{14}$.

Nótese que las afirmaciones están elegidas y enumeradas de manera que puedan ser contrapuestas las que ocupan el mismo lugar en una y otra enumeración, porque una de sus tesis centrales afirma, precisamente, que la decisión responsable de que las afirmaciones que ocupan el mismo lugar en una y otra enumeración resulten ser incompatibles entre sí, es la aceptación del siguiente enunciado:

- (4) Si las "totalidades» existen, entonces ellas tienen objetivos e intereses diferenciados por sí mismas (Agassi 1975: 147).

14 Las proposiciones típicamente defendidas por el institucionalismo y el psicologismo son, según el autor, las siguientes:

Institucionalismo.

(1.c).- La sociedad es la entidad social primaria (institucionalismo).

(2.c).- El deber primario de cada uno es con la sociedad propia (moralidad colectiva).

(3.c).- Las condiciones sociales influyen en y pueden alterar las condiciones individuales (colectivismo).

Psicologismo.

(1.d).- El individuo es la entidad social primaria (psicologismo).

(2.d).- La sociedad está sujeta a la crítica de la conciencia individual (autonomía de la moral).

(3.d).- Los individuos influyen en y pueden alterar las condiciones sociales (reforma institucional). 
Análogamente, sostiene que la decisión que trae consigo consecuencias análogas en relación con las (tres) proposiciones típicas del psicologismo y las (tres) típicas del institucionalismo es la aceptación de la frase siguiente:

- (5) O la sociedad es la entidad primaria, o lo es el individuo, pero no ambas a la vez (Agassi 1975: 149).

$Y$ considera que esas dos frase juntas (4 y 5), al referirse a objetivos o propósitos, aluden a las explicaciones finales o fundamentales en ciencia social, por lo que considera que pueden resumirse en y ser sustituidas por la frase de que

- $(\alpha)$ las explicaciones finales son las referidas a objetivos, que pueden ser individuales o sociales, pero no pueden ser ambas cosas a la vez (Agassi 1975: 151).

Por otra parte, la contraposición entre individualismo y holismo, y la que media entre psicologismo e institucionalismo, permiten que la combinación dos a dos de esas cuatro posiciones de lugar a otras cuatro posturas, de las cuales destaca dos como las más tradicionales. Por un lado, el individualismo psicologista, que sería la versión del individualismo propia del siglo dieciocho, y por el otro, el holismo institucionalista, que sería la versión propia del diecinueve, así como del Marxismo y del funcionalismo.

\section{El individualismo institucionalista}

El núcleo del análisis realizado por Agassi puede desdoblarse en dos partes simétricas complementarias. Por un lado, sostiene que la contraposición entre las versiones más tradicionales del individualismo y del holismo citadas, el individualismo psicologista y el colectivismo institucionalista respectivamente, descansa en la aceptación de las frases (4) y (5) expuestas más arriba, y que el autor propone resumir mediante la frase $(\alpha)$.

Por el otro, argumenta que si, por el contrario, se rechazan las frases (4) y (5) anteriores y se acepta consecuentemente la tesis individualista básica de que sólo los individuos tienen objetivos, propósitos e intereses, desparecen las contraposiciones entre las posiciones mencionadas y se enriquece consecuentemente las posibilidades explicativas basadas en las decisiones individuales. Llama «indivi- 
dualismo institucionalista» a la postura resultante. En ella, ni los grupos ni las instituciones sociales (que entiende de la manera más amplia, que «cubre de las costumbres a las constituciones, y de los vecindarios a los estados» [Agassi 1975: 152]) tienen objetivos ni intereses por sí mismos; sólo pueden llegar a tener los objetivos e intereses que los individuos les den, les adscriban ${ }^{15}$.

En definitiva, la propuesta de Agassi puede sintetizarse destacando dos puntos centrales. Por un lado, concentra la contraposición entre el individualismo y el holismo en dos tesis mantenidas por una y otra postura, respectivamente. La tesis individualista sostiene que sólo los individuos pueden tener objetivos, propósitos e intereses, mientras que la tesis holista es que los grupos y las instituciones sociales pueden también tener objetivos e intereses por sí mismos, con independencia de los que los individuos les confieran. En segundo lugar, trata de mostrar que si en relación con la postura a mantener se adoptan dos decisiones, desaparecen gran parte de los conflictos con otras tesis holistas y, lo que es más importante, se enriquece sustancialmente la capacidad explicativa individualista, al poder incorporar sin problemas en sus explicaciones factores sociales, y sin tener que condicionar su introducción a características como la de su reducibilidad, por ejemplo. La primera de esas decisiones es la de aceptar la tesis individualista básica (sólo los individuos pueden tener objetivos, propósitos e intereses). La segunda, la de rechazar que, entre los planos individual y colectivo o institucional, uno de ellos tenga que ser o considerarse el plano primario en detrimento del otro (con lo que se rechaza la tesis holista básica).

15 Nótese que al dejar de exigir que la entidad primaria sea o bien el individuo o bien la sociedad, pero no ambos a la vez, se admite que lo puedan ser los dos, «al menos en el sentido de que no podemos reducir la psicología a la sociología y no podemos reducir la sociología a la psicología" (Agassi 1975: 152). Asimismo, al rechazar las frases (4) y (5), se introduce una flexibilidad tal que hace compatibles las proposiciones típicas de las cuatro posiciones consideradas. En nuestro caso, el cambio más significativo es el que tiene lugar en relación con los factores afectan a las decisiones individuales. El autor expone el tema en estos términos: "Por lo tanto, en 2(a) no son los objetivos de las instituciones sino su existencia lo que afecta al comportamiento de los individuos: las instituciones existentes constituyen una parte de las circunstancias del individuo que junto con sus propósitos determinan su conducta de acuerdo con 2(b). Mientras que, de acuerdo con el individualismo psicologista, las condiciones materiales son las únicas que pueden considerarse relevantes, según el individualismo institucionalista la existencia de instituciones puede ser considerada también una circunstancia relevante. Esta adición enriquece 2(b) y, de ser el principio de racionalidad psicologista, la convierte en lo que Popper llama "lógica de la situación" (Agassi 1975: 153). 


\section{El adjetivo «metodológico»}

Para hacer justicia a la presentación original, resulta obligado añadir una referencia, por breve que sea, al énfasis con el que Agassi defiende la independencia de la perspectiva metodológica respecto de la ontológica y de la ética o política, y el carácter predominantemente heurístico de esa perspectiva que parece desprenderse de su exposición.

Después de definir sintéticamente las nociones de individualismo, psicologismo, colectivismo o holismo, e institucionalismo, trata de aclarar lo que para él significa el adjetivo "metodológico", "que puede acompañar a cualquiera de los cuatro términos definidos más arriban. Lo hace en referencia a Max Weber y sus conocidas tesis sobre el análisis sociológico, que debiendo basarse y centrarse en la conducta individual, no presupondría por parte del investigador, ni por parte de los individuos de las sociedades o colectivos investigados, la adopción de un sistema individualista de valores. Por el contrario, el método individualista no excluye que uno y otros puedan mantener puntos de vista holistas y aplicarlos. Lo característico de la perspectiva metodológica, según el autor, no reside en el plano de las tesis o hipótesis sustantivas, sino en que la defensa de la postura individualista se desarrolle mediante argumentos referentes al método (científico), "subrayando su fecundidad o su utilidad, etc.» (Agassi 1975: 145).

\section{En lugar de reducción, determinación por sobrevenida}

Como alternativa al requisito de la reducción, la literatura suele referirse a un concepto más moderno y menos exigente: la determinación por sobrevenida (supervenience), tomada de la filosofía de la mente. Hands (2001: 170) describe la noción de este modo:

La propiedad A sobreviene sobre la propiedad B si y sólo si B al fijar B se fija $A$, o en otras palabras, si no puede darse ninguna variación en $A$ sin una variación en B. De esta manera, si lo mental sobreviene sobre lo físico, eso quiere decir que no puede haber pensamientos diferentes sin procesos físicos diferentes; quedando de todos modos abierta la posibilidad de que procesos fisicos diferentes produzcan el mismo pensamiento ${ }^{16}$.

${ }^{16}$ Para más detalles, véase Kincaid 1998 b. 
La última cláusula se refiere al llamado problema de las multirrealizaciones, es decir, la posibilidad de que varias situaciones en el plano básico - neurológico, microeconómico- determinen una misma situación en el plano que sobreviene sobre el básico - mental, macroeconómico. El diferente tratamiento de ese problema suele señalarse como una de las diferencias cruciales entre reducción y sobrevenida (véase, por ejemplo, Kincaid 1998: 297).

Además, y como subraya Hoover (2001a: 68), la noción de sobrevenida garantiza que se puede usar racionalmente un lenguaje y unas categorías independientes para describir el nivel macro, sin dejar de subrayar la conexión o dependencia entre ambos niveles.

Las características indicadas del concepto de sobrevenida - determinación micro de los fenómenos macro, ausencia de reducción, relación de determinación del tipo muchos a uno, y autonomía del nivel que sobreviene- y la, a su juicio, clara irreducibilidad de la macroeconomía a la microeconomía llevan a Hoover (2001a: 73-4) a proponer ese concepto para reflejar más adecuadamente las relaciones entre la microeconomía y la macroeconomía. Sin embargo y con independencia de la ambigüedad del concepto, su compatibilidad con el IM es una cuestión sobre la que no hay acuerdo (véase, por ejemplo, Sensat, 1988: 192). Por ello, parece más indicado dirigir a la propuesta formulada por Agassi (después de haberlo hecho con la versión de Watkins) las preguntas como la que nos hacíamos al principio en relación con el modelo de Akerlof y Kranton.

\section{Comentarios finales}

\section{El carácter individualista del modelo de Akerlof y Kranton}

Cuando nos preguntábamos por el carácter individualista del modelo A-K en referencia a la versión de Watkins, surgían dudas y dificultades provenientes sobre todo del requisito de reducibilidad. Todas esas dudas y dificultades se desvanecen si para responder la misma pregunta recurrimos para al individualismo metodológico en la versión de Agassi. Según ésta, no hay nada en el modelo que aminore o deteriore su carácter individualista. El modelo se basa en la toma de decisiones por parte de los individuos, y en ningún momento se introducen colectivos o instituciones de los que se suponga que tienen objetivos, propósitos o intereses por sí mismos y con independencia de los que les confieran los indivi- 
duos que los integran. Por lo tanto, el modelo de A-K es, bajo el individualismo institucionalista y a todos los efectos, como los demás modelos habituales en teoría económica, que es al fin y a la postre lo que uno piensa ya desde la primera impresión. El individualismo institucionalista no establece ninguna diferencia entre el modelo de A-K y los modelos teóricos más habituales, por cuanto todos ellos se adecúan a esa posición igualmente bien.

Pero este carácter omniabarcante de esa versión del IM y la ausencia de distinciones entre las líneas de trabajo tan distintas como las que se desarrollan en el seno de la teoría económica, puede constituir un problema. Porque si, bajo una inspiración netamente popperiana, identificamos contenido informativo con capacidad de exclusión, ese carácter omniabarcante de la posición propugnada por Agassi nos lleva a tener que aceptar que revela, a cambio, una propuesta poco informativa ${ }^{17}$. No se puede decir que no cumpla su cometido. Al fin y al cabo, el IM nace como tal doctrina para oponerse a los planteamientos holistas y al análisis de Agassi le asiste indudablemente el mérito de poner en claro el núcleo de ese conflicto. Pero a cambio, tiene una capacidad discriminatoria muy pobre dentro del terreno de las construcciones que ese mismo criterio sanciona como (institucionalmente) individualistas.

\section{¿Un reenfoque más naturalista de la cuestión del individualismo?}

Estas apreciaciones vienen a reiterar algunas reflexiones que se produjeron en la sesión que la conferencia de la International Economic Association dedicó al en la teoría de juegos, a cuya ponencia presentada por Maarten Janssen (1998) ya nos hemos referido, igual que a dos de sus comentaristas, Ariel Rubinstein y Philippe Mongin.

Janssen (1998) aplica las nociones básicas propuestas por J. Agassi a la teoría de juegos con el propósito de estudiar las relaciones de esta teoría con el IM de manera más precisa, analizando diferentes vías seguidas en la propia teoría para justificar el equilibrio de Nash, y tratando de decidir hasta qué punto y con qué diferencias cumplen o satisfacen las nociones básicas susodichas.

17 Sensat (1988: 200) subraya la relación inversa entre el carácter restrictivo de una postura sobre individualismo y su plausibilidad. 
Por un lado y como nos ha sucedido a nostros en relación con el modelo de A-K, Janssen constata que la versión del individualismo institucionalista elimina las dificultades originadas por el requisito de reducibilidad a la hora de enjuiciar el papel de las instituciones como variables explicativas (Janssen 1998: 31 2, n. 4).

Pero esa flexibilidad se torna incapacidad y silencio analíticos cuando se trata de diferenciar las formas y modalidades de los desarrollos concretos que tienen lugar y pueden tenerlo bajo el enfoque individualista. Por ello, ya desde el principio de su artículo anuncia que su tercer objetivo es «argumentar [a los filósofos de la ciencia] que un examen más pormenorizado de la teoría de juegos pone de manifiesto que el individualismo institucionalista es una categoría que lo abarca todo [catch-all], y que se necesita un esfuerzo filosófico ulterior para hacer más precisa esa noción» (Janssen 1998: 2).

Asimismo, y en referencia a las líneas a las que ha pasado revista en su trabajo, orientadas a justificar el carácter individualista de las nociones básicas de la teoría de juegos, concluye:

Sin embargo, la estructura de las justificaciones estudiadas anteriormente es muy diferente de unos casos a otros, sucediendo además que la terminología existente relativa al individualismo institucionalista y al psicologista no puede dar razón de ellas. Este artículo puede leerse como un intento de estimular a los filósofos a redefinir versiones diferentes del IM a la luz de los desarrollos en las ciencias sociales en general y en la teoría de juegos en particular. (Janssen 1998: 26)

Philippe Mongin (1998: 51-52) se pronuncia en un sentido parecido al comentar el trabajo de Janssen. Por ejemplo, denuncia que, a pesar de los desarrollos teóricos registrados, la literatura sobre el IM haya seguido adoptando el planteamiento básico de las versiones más clásicas. Y resumen su punto de vista de esta forma tan contundente:

A lo largo del debate quizá se haya puesto de manifiesto el aspecto siguiente de la literatura sobre el IM: esta literatura está a una distancia conceptual tal de la teoría de juegos que no es nada fácil aplicarla en este campo. 
Por otra parte, esa distancia no se marca sólo con la teoría de juegos. Desde que se lanzan las propuestas austriacas clásicas sobre el individualismo metodológico, ha cambiado profundamente la misma forma de hacer y de entender la teoría económica. De manera mayoritaria, la teoría se desarrolla actualmente a base de construir y analizar modelos, que de manera cada vez más consciente se manejan como construcciones artificiales que no pretender recrear contrapartidas reales con algún grado suficiente de aproximación. En esa estrategia, el método individualista desempeña una función esencial. Hasta el punto de que no es fácil imaginar que esa forma de hacer la teoría se llevara a cabo con la misma intensidad sin ese apoyo metódico. Pero si esto es así, entonces el método individualista operaría en el contexto de descubrimiento tanto o más que en el de justificación, y con una función de carácter más instrumental y heurística. Sin embargo, en la literatura sobre el IM no es habitual encontrar menciones a estos temas. De hecho y como subraya Mongin, sorprende que a pesar de los años transcurridos, de la literatura dedicada al tema a lo largo de ellos, y de la propia evolución de la teoría de juegos y de la teoría económica a lo largo de ese largo periodo, las referencias básicas sobre el IM sigan siendo básicamente las mismas. Pero, además de sorprender, ese fenómeno revelaría que esa literatura ha seguido muy orientada por los propósitos y planteamientos filosóficos tan generales de esas referencias y de las demás propuestas clásicas, que estaban por otra parte especialmente condicionadas por su enfrentamiento con el materialismo histórico y con ideologías holistas como el fascismo y el nacionalismo.

\section{¿Es realmente individualista el método individualista?}

Además, estas apreciaciones conectan con otra característica intrigante en la literatura dedicada al IM: la escasa atención que se presta en las versiones de referencia a precisar si son o no individuales los agentes que toman las decisiones.

La cuestión es pertinente porque en la teoría económica, que es la teoría social individualista de referencia, quienes toman las decisiones no son habitualmente personas individuales. Son empresas, naciones, sindicatos, gobiernos, organizaciones. Incluso en el caso más cercano al individualista puro, el de los consumidores, la interpretación más habitual supone que los agentes son las unidades familiares ( $c f$. Kincaid 1998: 297; Janssen 1998: 4-5). En definitiva, las decisiones son tomadas individualmente por agentes, pero esos agentes que toman indi- 
vidualmente las decisiones no suelen ser individuos, sino entidades colectivas, $\operatorname{agregadas}^{18}$.

Naturalmente, el asunto es tan patente que, aunque no muy elaboradas, se han ido formulando algunas propuestas en la literatura para incluir expresamente en el enfoque individualista la posibilidad de que las decisiones sobre las que se basen las explicaciones no sean personas individuales. Por ejemplo, Janssen (1998: 4) propone el criterio siguiente:

Las economías domésticas, las empresas, los sindicatos y otras entidades pueden ser tratadas como "individuos" en tanto que las diferencias en propósitos e intereses de las personas que formen parte de esas entidades no parezcan ser importantes en relación con el problema en estudio. Por ejemplo, un sindicato puede ser considerado como una entidad "individual" en una explicación de los niveles salariales basada en el poder de negociación.

Propuestas como ésta, sin embargo, no evitan que el problema deba considerarse pendiente de un tratamiento que suscite un acuerdo amplio y claro. Debe señalarse, además, que la reducción de las decisiones colectivas a términos individuales puede no ser un asunto nada trivial, como sabemos después de los desarrollos registrados en la teoría de la elección social. Basta que una familia tenga tres miembros, por ejemplo, para que pueda presentarse la paradoja de la votación. En una comunidad de de copropietarios de una finca de viviendas puede presentarse con toda naturalidad un problema de manipulabilidad que dé lugar al conocido fenómeno del gorrón (free rider).

Adicionalmente, al ir admitiendo unidades de decisión no individuales para extender la cobertura del individualismo, éste puede ir quedando progresivamente vacío en el plano conceptual, como señalan, por ejemplo, Mongin (1998: 44), Kincaid (1998a: 297) y Uskali Mäki (apud Backhouse et al. 1998: 56-7).

En cualquier caso, subsiste la cuestión de por qué se ha destacado tradicionalmente tanto la dimensión individual, en detrimento de una consideración

18 Philip Mirowski (apud Backhouse et al. 1998: 59) pone de relieve el carácter colectivista de las aplicaciones de la teoría de juegos en la época de la Rand Corporation, que es la época en la que recibió el impulso decisivo y en la que los jugadores que importaban eran naciones, no personas individuales. 
más detenida de la variedad de agentes económicos. Sin duda, hay razones históricas mezcladas con el hecho de que la unidad de decisión más elemental y prototípica es la persona individual. Pero en este asunto también se podría estar revelando el trasfondo político que late en la motivación de las versiones clásicas del IM, trasfondo reforzado por el individualismo ontológico y al que nos referíamos ya al comienzo de estas páginas, al subrayar el propósito subyacente a esas versiones de sentar una base epistemológica y ontológica de crítica a opciones políticas e ideológicas colectivistas como, y sobre todo, el materialismo histórico, el nacionalsocialismo y el fascismo.

\section{Referencias}

AGASSI, Joseph, 1975, «Institutional individualism», British Journal of Sociology, v. 26, pp. 144-55.

AKERLOF, George, 1984, An economic theorist's book of tales, Cambridge University Press, Cambridge.

AKERLOF, George A. y KRANTON, Rachel E., 2000, «Economics and Identity», Quarterly Journal of Economics, v. 115, n. 3, pp. 715-53.

ARROw, Kenneth J., 1994, "Methodological Individualism and Social Knowledge». American Economic Review (Papers and Proceedings), v. 84, n. 2, pp. 3-9.

Backhouse, Roger E., Hausman, Daniel M., Mäki, Uskali Y Salanti, Andrea (eds.), 1998, Economics and Methodology. Crossing Boundaries, Macmillan Press Led, Londres, y St. Martin's Press, Inc., N. York.

BlaUG, Mark, 1992, The methodology of economics, or how economists explain, Cambridge University Press, Cambridge.

DAVIS, John B., 2004, The Theory of the Individual in Economics: Identity and Value, Routledge \& Keagan Paul, Londres.

DaVIS, John B., HaNDS, D. Wade y MäKI, UsKali, eds., 1998, The Handbook of Economic Methodology, Edward Elgar, Cheltenham (UK)-Northampton (MA, USA).

ELSTER, Jon, 1982, "Marxism, Functionalism, and Game Theory», Theory and Society, v. 11 , pp. $453-82$.

HANDS, D. Wade, 2001, Reflection without Rules. Economic Methodology and Contemporary Science Theory, Cambridge University Press, Cambridge. 
Hausman, Daniel M., 1992, The Inexact and Separate Science of Economics, Cambridge University Press, Cambridge.

Hausman, Daniel M., 2003, "Taking causal questions seriously" [Reseña de Hoover 2001 a y 2001b], Journal of Economic Methodology, v. 10, n. 2, pp. 259-69.

HOOVER, Kevin D., 2001a, The Methodology of Empirical Macroeconomics, Cambridge University Press, Cambridge.

HoOver, Kevin D., 2001b, Causality in Macroeconomics. Cambridge University Press, Cambridge.

JANSSEN, Maarten, 1998, «Individualism and Equilibrium Coordination in Games», en Backhouse et al., 1998: 1-35.

KINCAID, Harold, 1998, «Methodological Individualism/Atomism», en Davis et al. 1998: 294-300.

MongIN, Philippe, 1998, «Comment: Individualistic Explanation and Strategic Justification", en Backhouse et al., 1998: 42-53.

Nagel, Ernest, 1961, The Structure of Science. Problems in the Logic of Scientific Explanation, Routledge \& Kegan Paul, Londres.

POPPER, Karl R., 1957, The Poverty of Historicism. Londres, Routledge and Keagan Paul. (Las citas están tomadas de la versión castellana publicada por Taurus Ediciones, S.A. en Madrid en 1961, bajo el título La miseria del historicismo).

ROEMER, John E., 1982, "Methodological Individualism and Deductive Marxism", Theory and Society, v. 11, pp. 513-20.

RUBinSteIN, Ariel, 1998, “Comment», en Backhouse et al., 1998: 36-41.

SCHUMPETER, Joseph A., 1967, History of Economic Analysis, George Allen \& Unwin Ltd, Londres.

SCHWARTZ, Pedro, 1970, «El individualismo metodológico y los historiadores», en Ensayos de Filosofia de la Ciencia. En torno a la obra de Sir Karl R. Popper, Tecnos, Madrid, pp. 117-40.

SENSAT, JULIUS, 1988, "Methodological Individualism and Marxism», Economics and Philosophy, v. 4, pp. 189-219.

VISKOVATOFF, Alex, 1998, «Holism», en Davis et al. 1998: 229-32. 
WaTKINS, J. W. N., 1968, "Methodological Individualism and Social Tendencies", en BrodbeCK, May (ed.), Readings in the Philosophy of the Social Sciences, Collier-Macmillan Ltd., Londres, y The Macmillan Company, N. York.

ZAPATERO MARTIN, Juan Carlos, 1977, «K. Popper y la metodología de las ciencias sociales", Cuadernos Económicos de ICE, n. 3-4, pp. 97-109. 